\title{
磑化铯薄膜光阴极的研究进展
}

\author{
焚 龙，黎宇坤，陈蹈，李 晋，杨志文，袁铮，邓 博，曹柱荣，胡 昕 \\ (中国工程物理研究院激光聚变研究中心, 绵阳 621900)
}

摘 要: 碘化铯(CsI)薄膜因对 X 射线及紫外光具有高的光电转换效率而倍受关注。在核物理、高能物理以及天体物 理研究的推动下, 研制高量子效率 $(\mathrm{QE}) 、$ 性能稳定的 CsI 薄膜光阴极成为了近年来研究的热点。然而, 目前人们对 某些影响其性能的因素还不完全清楚或确定。本文综述了 CsI 薄膜光阴极的最新研究进展, 总结了影响 QE 的因素 和光阴极的老化机理，重点关注了一些存在争议的问题，并对其研究发展方向进行了探讨。

关 键 词: CsI 薄膜; 光阴极; 量子效率; 受潮老化; 辐照老化; 综述

中图分类号: TQ174 文献标识码: A

\section{Recent Progress in Research on CsI Thin Film Photocathodes}

FAN Long, LI Yu-Kun, CHEN Tao, LI Jin, YANG Zhi-Wen, YUAN Zheng, DENG Bo, CAO Zhu-Rong, HU Xin (Research Center of Laser Fusion, China Academy of Engineering Physics, Mianyang 621900, China)

\begin{abstract}
Caesium iodide (CsI) thin films have attracted much attention due to good electron transport and emission properties in the soft X-ray and extreme ultraviolet range. With the rapid development of nuclear physics, high energy physics and astrophysics, the requirements for CsI film photocathodes become increasingly severe, and it is therefore of considerable interest to develop CsI photocathodes with high quantum efficiency (QE) and stable performance. However, the internal mechanisms of QE variation and photocathode aging are not entirely clear or certain up to date. In this paper, the recent progress on CsI film photocathodes is reviewed and some controversial issues are introduced. Several perspectives are also proposed in the end.
\end{abstract}

Key words: CsI film; photocathode; quantum efficiency; moisture aging; irradiation aging; review

CsI 属 B2 型 $\mathrm{CsCl}$ 结构, 禁带宽度 5.9 6.2 eV, 电 子亲和势 $0.1 \sim 0.2 \mathrm{eV}$, 不同研究者测得二次电子逃逸 长度在 10 34 nm 之间, 取决于入射光子能量、二次电 子能量和材料制备工艺。大的二次电子逃逸长度和低 的电子亲和势使 CSI 对软 X 射线、极端紫外光和远紫 外光具有高的光电转换效率, 另外, CsI 光阴极二次电 子能量分布较窄, 对制备和实际应用环境的要求相对 较底。这些性质使其在核物理、高能物理以及天体物 理研究中具有重要的应用。美国国家点火装置(NIF) 使用的条纹相机、欧洲大型强子对撞机使用的环成像
切伦科夫探测器采用了 CsI 薄膜作为光阴极，国内开 展的激光惯性约束聚变研究使用的 X 射线条纹相机、 暗物质探索实验使用的气体电子倍增器也采用了 CsI 薄膜作为光电转换的核心元件 ${ }^{[1-5]}$ 。CsI 薄膜光阴极按 光电流逸入真空界面的不同分为透射式和反射式两 种, 图 1 为两种结构的示意图。

尽管 CsI 及掺杂激活材料的闪㷧发光性质得到 了人们深入的研究, 但对其光电转换及发射性能的 研究却相对滞后。近年来, 随着激光惯性约束聚变、 高能粒子探测以及天体物理研究的快速发展, 人们

收稿日期：2014-07-02; 收到修改稿日期：2014-09-03

基金项目: 国家自然科学基金(10905050); 中国工程物理研究院发展基金(2013B0102011) National Natural Science Foundation of China (10905050); Development Foundation of China Academy of Engineering Physics (2013B0102011)

作者简介: 焚 龙(1985-), 男, 博士, 助理研究员. E-mail:sfanlong@163.com 

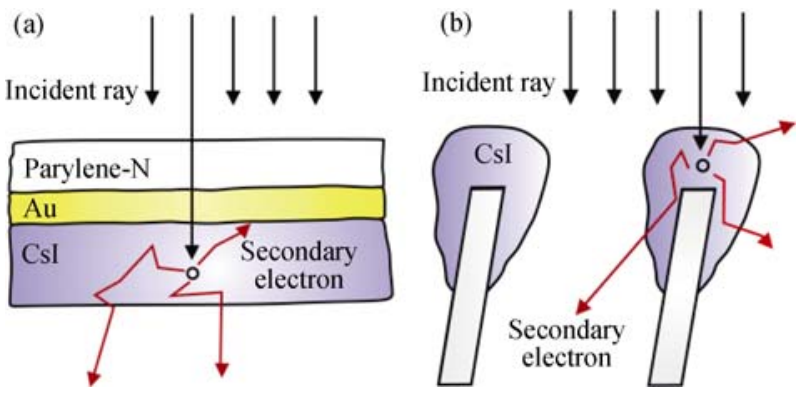

图 1 (a) Parylene-N / Au /CsI 透射式光阴极; (b) MCP 表面反 射式 CsI 光阴极

Fig. 1 (a) Parylene-N /Au /CsI semitransparence photocathode; (b) Refective CsI photocathode coated on the surface of microchannel plate (MCP)

对 CsI 光阴极提出了越来越高的要求。多个研究小 组探索发展了 CsI 薄膜光阴极的制备方法, 研究了 薄膜结构、表面状态、基底结构等因素对 QE 的影 响, 并对光阴极老化机制和防护方法进行了探讨。 然而, 众多研究者 ${ }^{[6-13]}$ 指出, 某些影响 CsI 薄膜光阴 极效率的因素以及老化机理尚不完全清楚或确定。 本文介绍了近年来国内外 CsI 薄膜光阴极的研究进 展, 试图对影响量子效率(QE)的因素及老化机理进 行总结, 重点介绍了一些目前还存在争议的问题, 最后对 CsI 薄膜光阴极的研究方向进行了展望。

\section{1 影响 QE 的因素}

QE 是 CsI 薄膜光阴极最重要的性能参数, 研究 各种影响 $\mathrm{QE}$ 的因素以及相应的物理机制是制备高 性能光阴极的关键和工艺改进的理论基础, 因而引 起了研究者极大的兴趣。CsI 薄膜的 QE 与制备方法、 制备环境、膜厚、缺陷、表面污染及基底等都有密 切关系, 在实际应用中受探测器结构、工作波段、气 体环境及电场等因素影响。本文针对薄膜制备中影 响 QE 的因素以及相应的研究进展作简单介绍。

\section{1 制备方法}

制备 CsI 薄膜光阴极的传统方法为真空热蒸发 法, 目前针对制备工艺的改进大多以热蒸发法为 基础。一些研究也采用电子束蒸发法制备 CsI 光阴 极 ${ }^{[3,14-15]}$ 。Schyns ${ }^{[16]}$ 及 $X i e^{[6,17]}$ 等的研究表明, 这两 种方法制备的 CsI 光阴极 $\mathrm{QE}$ 相差不大, 但电子束蒸 发法制备的薄膜颗粒较小、结构更致密, 在空气中 具有较好的稳定性。近年来, 人们探索了离子束溅 射法和激光等离子体沉积法等方法。2005 年, Nitti 等 ${ }^{[18-19]}$ 首次采用离子束溅射法在抛光石英基底上制 备了 $100 \mathrm{~nm}$ 的 CsI 光阴极, 导电层为 $\mathrm{Ti} / \mathrm{Au}$, 采用不 同溅射能量得到的薄膜表面 $\mathrm{Cs} / \mathrm{I}$ 元素比例不同, 相
比于真空热蒸发法, 得到的薄膜更致密、粗鉛度更 小, 但 QE 值却相对较低, 他们将原因归因于表面 发射面积的降低。2011 年, Fairchild 等 ${ }^{[20]}$ 采用激光 脉冲沉积法(PLD)在 Si 基底上制备了 CsI 薄膜, 薄膜 中存在 $\mathrm{Cs}_{2} \mathrm{O}$ 及 $\mathrm{CsIO}_{3}$ 等杂相。2012 年, Brendel 等 ${ }^{[21]}$ 采用 PLD 法制备了 CsI 光阴极, QE 值与热蒸发法相 近, 采用激光束扫描发现, PLD 法制备的光阴极光电 发射均匀性变化在 20\%以内, 而热蒸发法制备的 CsI 薄膜变化达到了 $100 \%$, 另外, 与基底的附着力以及 在空气中的稳定性都要优于热蒸发法, 他们指出 PLD 法是一种有前途的 CsI 薄膜光阴极制备方法。

\section{2 制备环境}

保证制备环境的洁净是制备高效光阴极的前提。 对于 CsI 薄膜光阴极, 尽管一些研究者 ${ }^{[7,22]}$ 并未发现 真空度的提高对 QE 有明显的影响, 但 Hoedlmoser 等 ${ }^{[23]}$ 认为, 真空系统中(真空度大于 $10^{-5} \mathrm{~Pa}$ )残余水分 子与任何表面的碰撞概率为 $0.1 \sim 1$ 分子层/s, 当 CsI 薄膜以 $1 \mathrm{~nm} / \mathrm{s}$ 的速率生长时(约 2.2 分子层 $/ \mathrm{s}$ ), 会有 相当多的水分子进入薄膜中, 导致刚制备的 CsI 薄 膜 QE 不高, 另外, 蒸发容器壁上沉积的 CsI 层数及 清理次数对实验结果也有影响。Breskin ${ }^{[24]}$ 和 Tremsin ${ }^{[7]}$ 等指出环境清洁度及 CsI 源纯度的不同可 能是一些研究者得到不同甚至矛盾实验结果的原 因。Friese 等 ${ }^{[25]}$ 的研究结果表明, 不充分清洁的基 底可能会使 QE 下降 30\%。

\section{3 膜厚}

由于光子吸收以及二次电子传输等性质与 CsI 薄膜厚度直接相关, 对光阴极厚度的理论设计与 实验研究受到了人们广泛关注。对于透射式 CsI 光 阴极而言, QE 对厚度变化敏感。Valentini 等 ${ }^{[26]}$ 的计 算结果表明, 对于波长为 $170 \mathrm{~nm}$ 的光子, $10 \mathrm{~nm}$ 厚 度的 CsI 薄膜具有最大的 $\mathrm{QE}$ ，当厚度为 $60 \mathrm{~nm}$ 时 $\mathrm{QE}$ 已经非常低。 $\mathrm{Lu}^{[27]}$ 和 Boutboul ${ }^{[28]}$ 等认为用于 $160 \sim 190 \mathrm{~nm}$ 波段 CsI 光阴极的最优厚度为 10 20 nm, 但在实验中发现 $20 \mathrm{~nm}$ 厚的 CsI 薄膜暴露在空气 中仅几分钟就发生了破裂。我们课题组建立了软 $\mathrm{X}$ 光能区 $(0.1 \sim 10 \mathrm{keV}) \mathrm{CsI}$ 二次电子产出与膜厚及 入射角的关系，并采用同步辐射光源测试了透射 式光阴极谱响应灵敏度, 结果表明最优厚度约为 $60 \mathrm{~nm}^{[29-31]}$ 。对于反射式 CsI 薄膜光阴极, QE 在膜 厚为几十 $\mathrm{nm}$ 时达到最大值 ${ }^{[32-33]}$, 并且在一定范围 内, 厚度增加对 $\mathrm{QE}$ 的影响不大, 大于 $500 \mathrm{~nm}$ 的膜厚 会使电场作用减弱导致 QE 下降 ${ }^{[17]}$ 。Braem 等 ${ }^{[32]}$ 指 出, 厚度大于 $60 \mathrm{~nm}$ 的 CsI 薄膜虽不能产生更高的 $\mathrm{QE}$, 但是却能在基底上形成更均匀的薄膜, 对空 气耐受性也更好。由于过薄的 CsI 薄膜稳定性与均 
匀性并不理想, 某些 CsI 光阴极的实际设计和制备 选择了更大的厚度, 放弃了一定的 QE 以获得更好 的稳定性。

\section{4 缺陷及表面污染}

特定波段 CsI 薄膜膜厚与 QE 关系的半经验模型 通常由光电流发射的实验数据拟合得到, 这些模型将 二次电子传输过程中能量的损失归于电子与声子的 散射。而在实际传输过程中, 除与晶格热振动散射外, 薄膜中的杂质 ${ }^{[7]}$ 、色心 ${ }^{[34]}$ 、位错 ${ }^{[23]}$ 、颗粒内部和边界 中的水分子以及颗粒界面 ${ }^{[35]}$ 等都可能使电子在传输 中损失能量; 薄膜表面的吸附水 ${ }^{[13]} 、 \mathrm{O}_{2}{ }^{[36]} 、 \mathrm{C}$ 元素及 化合物 ${ }^{[6,9,11,37]}$ 以及 Cs、I 元素比例的变化和相应化 合物的生成 ${ }^{[14]}$ 都可能造成电子亲和势增大, 阻碍电 子向真空发射。

目前, 人们对 CsI 薄膜中缺陷及表面污染产生 的原因及影响 $\mathrm{QE}$ 的原理还存在不同的看法。薄膜 在生长过程中可能会受到镀膜系统中残余水分子、 碳氢化合物以及挥发 I 元素的污染 ${ }^{[12,23,38]}$, 并可能 产生色心及 Cs 析出 ${ }^{[38-39]}$ 。受潮和辐照引起的缺陷等 将在第 2 节中具体讨论。

\section{5 薄膜结构}

CsI 薄膜表面形貌、疏密程度以及颗粒大小等 是影响 QE 的重要因素。一些研究表明, 通过控制 镀膜真空度及充入 $\mathrm{Ar}$, 制备的变密度结构以及具有 线状结构的 CsI 薄膜具有更高的 $\mathrm{QE}^{[40]}$ 。Nitti 等 ${ }^{[18]}$ 的实验结果表明, 无论是热蒸发还是离子束溅射制 备的 CsI 薄膜, QE 随薄膜表面粗粘度增大而上升。 对于薄膜中颗粒大小的影响, 他们认为阴极老化导 致的薄膜表面结构变化(小颗粒变大完全改变了薄 膜的表面结构)会引起 $\mathrm{QE}$ 下降 ${ }^{[18]}$, 由基底引起的小 颗粒长大受阻是 $\mathrm{QE}$ 下降较小的重要原因 ${ }^{[41]}$ 。另一 些研究者认为, 较小的颗粒会减小 CsI 光阴极的 $Q E$, 原因是更多的颗粒界面影响了二次电子的传输和发 射 ${ }^{[23,35,42]}$ 。
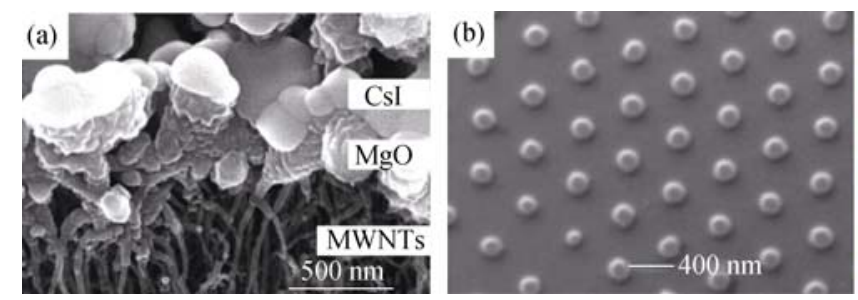

\section{6 基底材料及结构}

CsI 薄膜光阴极的基底材料及结构一直是人们 研究的重点。Friese 等 ${ }^{[25]}$ 发现以导电石墨层为基底 的 CsI 薄膜光阴极在多天保存后 $\mathrm{QE}$ 出现了连续的 上升。Singh ${ }^{[43]}$ 和 Valentini ${ }^{[26]}$ 等的研究结果表明对 $\mathrm{UV}$ 波段高反射率的 $\mathrm{Al}$ 基底可以提高光阴极的 $\mathrm{QE}$, 原因是反射导致光子吸收长度增加。

近年来, 微结构基底成为了 CsI 薄膜光阴极新 的研究热点。Halvorson 等 ${ }^{[44]}$ 以光刻模板法制备的楔 形微结构 Parylene/Au 自支撑膜作为基底, 使得透射 式 CsI 阴极光电子产出提高了近 5 倍, 增益来源于光 子吸收长度和光电发射面积的增加以及二次电子行 程的减少。Lee 等 ${ }^{[45]}$ 将多壁碳纳米管(MWCNTs)的场 致发射特性与 CsI 的光电发射性质相结合, 使 $\mathrm{CsI} / \mathrm{MgO} / \mathrm{MWCNTs} / \mathrm{Si}$ 结构的薄膜光阴极在 $147 \mathrm{~nm}$ 处 的光电流较 $\mathrm{CsI} / \mathrm{Si}$ 结构光阴极上升了几个量级, 但 CsI 在场致发射条件下的稳定性仍是一个问题 ${ }^{[46]}$ 。

Nitti 等 ${ }^{[41]}$ 在等离子体刻蚀过具有微结构的 $\mathrm{Si}$ 和不锈 钢基片上生长了 CsI 薄膜, 尽管 $\mathrm{QE}$ 值与以 PCB 板为 基底的 CsI 光阴极类似，但暴露于 $45 \%$ 湿度的空气 $24 \mathrm{~h}$ 后, 在 $150 \mathrm{~nm}$ 处的 QE 仅下降了 $30 \%$, 而以传统材料 为基底的光阴极 $\mathrm{QE}$ 却下降了 $70 \%$ 。图 2 为 $\mathrm{CsI} / \mathrm{MgO} / \mathrm{MWCNTs} / \mathrm{Si}$ 结构光阴极和等离子体刻蚀过 的 $\mathrm{Si}$ 基底的扫描电镜(SEM)照片以及 $\mathrm{Si}$ 基底上生长的 CsI 薄膜暴露空气前后原子力显微镜(AFM)照片 ${ }^{[41,45]}$ 。

\section{2 老化研究}

在 CsI 薄膜光阴极的研究与应用中, 稳定性始 终是研究者面临的一个重要技术难题。由于某些设 备的实际应用与操作使 CsI 光阴极不可避免的接触 空气, 由此导致的性能不稳定制约了其应用 ${ }^{[25,47]}$ 。 另外, CsI 光阴极在一定强度的 X 射线或紫外光中工 作，辐照环境也会导致 QE 下降。目前，人们对 CsI

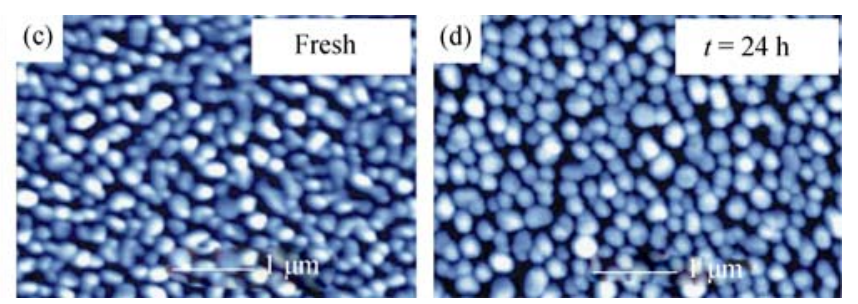

图 2 (a) $\mathrm{CsI} / \mathrm{MgO} / \mathrm{MWCNTs} / \mathrm{Si}$ 结构光阴极的 SEM 照片 ${ }^{[45]}$; (b)等离子体刻蚀过的 Si 基底的 SEM 照片; (c)Si 基底上 CsI 薄膜暴 露于空气前的 AFM 照片; (d) Si 基底上 CsI 薄膜暴露于空气 $24 \mathrm{~h}$ 的 AFM 照片 ${ }^{[41]}$

Fig. 2 (a) SEM image of the CsI/ MgO/ MWCNTs/ Si photocathode ${ }^{[45]}$; (b) SEM image of the Si substrate patterned by colloidal lithography after lift-off process; (c)AFM image of the fresh CsI film evaporated on the Si patterned substrate; (d) AFM image of the CsI film on the Si patterned substrate exposed to ambient air for $24 \mathrm{~h}^{[41]}$ 
薄膜光阴极的老化过程与相应的物理机制还缺乏系 统和深入的理解, 而这正是研制高性能光阴极必需 的理论基础。研究 CsI 薄膜在潮湿空气和辐照环境 中的老化机理, 探索各种条件下 $\mathrm{QE}$ 衰减的原因成为 了辐射探测研究与材料科学交叉领域的当务之急。

\section{1 受潮老化}

由于 $\mathrm{Cs}$ 和 $\mathrm{I}$ 电负性差异较大 $(\mathrm{Cs}$ 为 0.79 而 $\mathrm{I}$ 为 2.66), 使 CsI 表现出较强的极性, 极易吸附同样具 有极性的水分子并形成离子偶极键. 暴露空气会使 CsI 薄膜光阴极的效率下降, 在极端潮湿的环境中 (如呼吸), CsI 薄膜表面会变为乳白色 ${ }^{[6,48]}$, 效率完 全被破坏。图 3 为刚制备、在空气中暴露后、极端 潮湿环境中的 CsI 薄膜的 SEM 照片以及 NIF 使用的 $X$ 射线条纹相机上的透射式 CsI 光阴极信号下降与 空气暴露时间的关系 ${ }^{[47,49]}$ 。

目前人们对受潮导致光阴极 $\mathrm{QE}$ 下降有几种解 释。Hoedlmoser 等 ${ }^{[23]}$ 认为潮湿空气中 CsI 薄膜表面 吸附的水层(溶液层)使到达薄膜表面的光电子能量 降低, 并将热处理对长波段 $\mathrm{QE}$ 提高更明显的原因 归因于表面吸附水层厚度的影响。Triloki 等 ${ }^{[50]}$ 认为 QE 下降是水扩散进入 CsI 薄膜内部产生的缺陷影 响了电子的传输。一些研究者 ${ }^{[6,37]}$ 发现受潮后薄膜 表面 Cs 含量偏高, 将原因归于 CsI 与水的反应和 $\mathrm{HI}$ 的挥发:

$$
\mathrm{CsI}+\mathrm{H}_{2} \mathrm{O} \rightarrow \mathrm{CsOH}+\mathrm{HI} \uparrow
$$

Xie 等 ${ }^{[6]}$ 认为这可能是 $\mathrm{QE}$ 下降的原因之一。
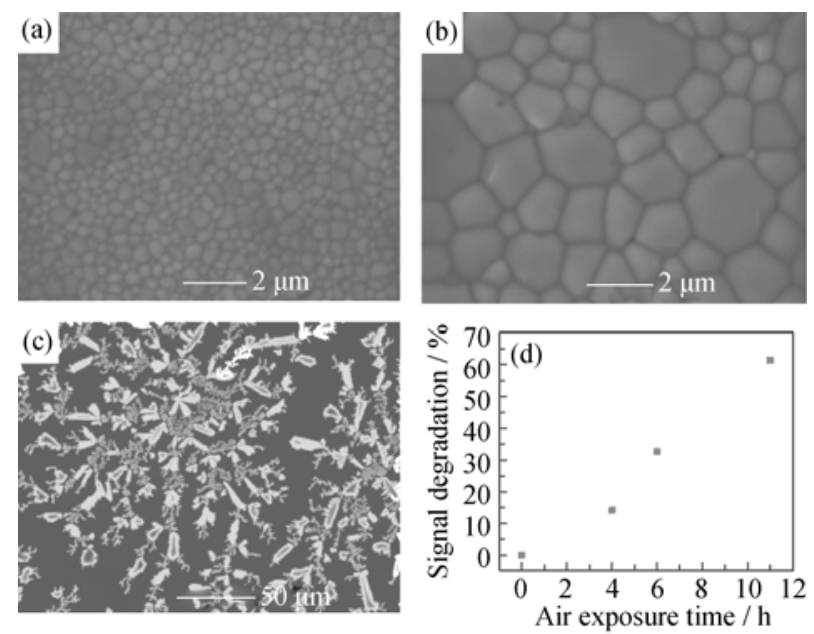

图 3 (a)刚制备、(b)在空气中暴露后、(c)在极端潮湿环境中 暴露后 CsI 薄膜的 SEM 照片 ${ }^{[49]}$; (d)NIF 采用的 X 射线条纹相 机上 $200 \mathrm{~nm}$ 厚的 CsI 透射式光阴极信号下降与空气暴露时间 的关系 ${ }^{[47]}$

Fig. 3 SEM images of as-deposited (a), aged in ambient air (b) and extreme moisture exposed CsI films (c) ${ }^{[49]}$; Percent degradation in the yield signal of streak camera used at NIF from a $200 \mathrm{~nm}$ thick CsI cathode as a function of air exposure time ${ }^{[47]}$
Kumar 等 ${ }^{[39,51]}$ 的实验结果表明 Cs 的含量仅在部分 微区域偏高, 他们认为 Cs 含量在真空热蒸发一开 始就出现增加, 原因为 I 的升华和色心聚集与析出。 一些研究者认为受潮导致的薄膜形貌及颗粒尺寸的 变化对 $\mathrm{QE}$ 也有影响 ${ }^{[2,11,18,35]}$, 其中 Nitti 等 ${ }^{[18]}$ 认为这 是 $\mathrm{QE}$ 下降的重要原因。

几乎所有对 CsI 薄膜光阴极受潮的研究都发现薄 膜中颗粒尺寸变大, 但对颗粒生长的动力学过程有不 同的描述: Xie 等 $^{[6]}$ 认为, 薄膜中较小颗粒在颗粒顶端 岛状水溶液重力、静电引力及表面张力作用下发生合 并形成大颗粒; Triloki ${ }^{[50]}$ 和 Boutboul ${ }^{[28]}$ 等认为颗粒长大 源于薄膜表面溶液相中水分挥发所引起的再结晶; Hoedlmoser 等 ${ }^{[23]}$ 认为, 薄膜中水分子的存在降低了 CsI 的扩散激活能, 使得扩散加速, 从而使颗粒长大, 笔者 进一步认为水在颗粒间界的 “短路扩散” 效应在颗粒 的生长中起了重要作用 ${ }^{[99]}$ 。对于极端潮湿空气中 CsI 薄膜表面变为乳白色的原因, Tremsin 等 ${ }^{[48]}$ 认为是 CsI 颗粒团聚以及大量基底暴露所造成, Xie 等 ${ }^{\left[{ }^{6}\right]}$ 认为可能 是 $\mathrm{CsOH}$ 溶解于水中所造成。

刚制备的 CsI 薄膜光阴极的 QE 并不理想, 经真 空退火处理后, QE 有一定程度的上升并能减缓在空 气中的老化, 对于有限度暴露空气造成的 QE 下降, 也可通过真空退火进行恢复，而在极端潮湿环境中 造成的 QE下降却不可能恢复 ${ }^{[52]}$ 。尽管研究众多, 但 人们对于真空退火法的原理还存在争议。Boutboul 等 ${ }^{[53]}$ 的实验结果表明退火并未增加 CsI 薄膜中二次 电子的逃逸长度, 认为 $\mathrm{QE}$ 的上升源于薄膜表面电 子的发射性能的增强, 另一些研究者 ${ }^{[34,54]}$ 认为电子 传输性质的改变也应考虑在内。Hoedlmoser 等 ${ }^{[23]}$ 认为退火导致的 $\mathrm{QE}$ 上升是 CsI 薄膜结构变化与脱 水共同作用的结果：刚制备的 CsI 薄膜中含有水分 (即使在 $10^{-5} \mathrm{~Pa}$ 的真空度下), 真空退火使水分从薄 膜内部扩散到表面并挥发, 在这个过程中薄膜结构 发生了变化(颗粒长大), 表面吸附的水分也被除去。 而 Buzulutskov 等 ${ }^{[12-22]}$ 则认为 Hoedlmoser 等对于去 除表面水分的解释并不完全合理，理由是真空退火 对 $\mathrm{NaI}$ 和 $\mathrm{CuI}$ 阴极也有类似的效果，而这几种化合 物在水中的溶解度相差了 5 个数量级, 他们认为最 有可能的解释是加热导致的薄膜中 $\mathrm{CsI}_{3}$ 和 $\mathrm{CsI}_{5}$ 等杂 相的分解。而 Baishali 等 ${ }^{[35]}$ 的实验结果表明, 仅将 暴露过空气(70\%湿度, $1 \mathrm{~h}$ 暴露时间)的 CsI 薄膜进行 真空 $\left(\right.$ 约 $\left.10^{-4} \mathrm{~Pa}\right)$ 脱水处理, 同样可使 $\mathrm{QE}$ 得到恢复。

除退火以外，人们还探索了一些其它方法来减少 受潮对 CsI 薄膜光阴极 QE 的影响。一些研究表明在 CsI 薄膜表面蒸发一层极薄的 LiF 或 NaF 层(厚度小于 
$3 \mathrm{~nm}$ ), 暴露空气后光阴极 $\mathrm{QE}$ 出现了上升, 原因为 $\mathrm{LiF}$ 或 $\mathrm{NaF}$ 吸附水后电子亲和势出现了下降 ${ }^{[55]}$ 。一些研究 发现, 在热蒸发时给基底加上负的偏压, 能够制备出 更不容易受潮的 CsI 光阴极, 原因为薄膜表面有更多 的 $\mathrm{I}^{-}$离子, 倾向与水分子中 $\mathrm{OH}$ 共价键的 $\mathrm{H}$ 端形成偶 极层, 使电子亲和势下降 ${ }^{[37,43]}$ 。有研究表明相比于慢 的蒸发速率, 较快的蒸发速率制备的 CsI 薄膜更不容 易受潮, 但并未给出明确的解释 ${ }^{[56-57]}$ 。

\section{2 辐照老化}

由于 CsI 薄膜光阴极在正常工作环境中老化过 程比较缓慢, 对辐照老化的研究一般采用了加大剂 量、加速老化的方法。对于特定剂量的辐射, 某些 情况下加大辐射强度而 QE 值反而下降较慢, 这使 得对辐照老化的研究变得更为复杂, 并与实际老化 过程有一定差异 ${ }^{[7,58]}$ 。

人们对辐照老化机理有几种解释。Breskin 等 ${ }^{[24]}$ 将 CsI 薄膜光阴极在辐照中效率下降归因于 CsI 的 光解和表面 I 的挥发, 表示为:

$$
\mathrm{h} v+\mathrm{CsI} \rightarrow \mathrm{Cs}^{+}+\mathrm{I}+\mathrm{e}^{-}
$$

一些研究者 ${ }^{[7,20,34,59-60]}$ 认为辐照导致 CsI 薄膜中色 心的产生、扩散与合并是光阴极老化的原因: 辐照 形成的 $\mathrm{F}$ 心、 $\mathrm{H}$ 心、聚集 $\mathrm{F}$ 心及聚集 $\mathrm{H}$ 心等色心在 禁带中引入新的能级, 改变光的吸收性质并影响电 子的传输, 另一方面, 色心等缺陷可能从薄膜内部 扩散出来, 在阴极表面形成 Cs 金属岛, 而 I 的含量 出现下降甚至消失, 使得薄膜表面光电子发射性能 受到影响。另一种解释认为, 当 CsI 薄膜暴露于辐 照中, 光电流将 $\mathrm{Cs}^{+}$离子带到基底并与基底发生反 应，同时 $\mathrm{I}^{-}$离子被带到了阴极表面，I离子的聚集使 $\mathrm{QE}$ 下降并改变薄膜的电阻率 ${ }^{[61]}$ 。Singh 等 ${ }^{[9,11]}$ 认为 晶格结构的逐层破坏和薄膜表面 C 钝化层的形成可 能是 UV 辐照导致光阴极老化的原因, 他们的实验
结果表明 CsI 薄膜表面的 Cs 和 I 元素比例在辐照后 并未发生变化, 而 C 含量出现上升, 认为 UV 辐照使 CsI 薄膜的结构从表面开始逐层受到破坏, 影响了电 子传输性能, 另外, CsI 颗粒顶端形成了 C 钝化层, 光 电子从颗粒边界向外发射。另一些研究者认为 CsI 颗粒边界处无光电子产出, 是二次电子发射的 “死

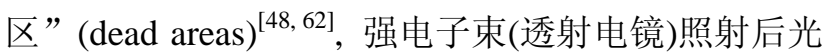
阴极的退化最先发生在颗粒边界处 ${ }^{[48,50]}$ 。图 4 中(a)、 (b)为经 $5 \mathrm{~d} \mathrm{UV}$ 辐照后(160 nm 波长)CsI 薄膜的 AFM 照片和光电子显微镜(PEEM)照片 ${ }^{[11]}$, Singh 等认为 PEEM 照片中暗区为颗粒顶端的 C 钝化层, 光电子 从亮区颗粒边界处向外发射; 图 4(c)为 UV 辐照后 (253.7 $\mathrm{nm}$ 波长, $10^{15}$ 光子 $\left./ \mathrm{mm}^{2}\right) \mathrm{CsI}$ 薄膜的透射电镜 (TEM)照片 ${ }^{[48]}$, Tremsin 等认为颗粒边界处无二次电 子发射, 边界处亮区的白点为电子束引起的微孔洞, UV 辐照过的光阴极在边界处退化较快。

另外, 一些研究表明退火以及加偏压能够降低辐 照对 CsI 薄膜 QE 的影响 ${ }^{[60,63]}$ 。有研究者观察到辐照 后薄膜中颗粒尺寸变大 ${ }^{[9,11]}$ 。应指出的是,一些辐照老 化实验的过程中有短暂的空气暴露 $[11,34,48,60,63]$ 。

\section{3 总结与展望}

核物理、高能物理以及天体物理等学科的快速 发展对 CsI 薄膜光阴极提出了越来越高的要求, 然 而目前人们对影响光阴极性能的某些因素还未完全 清楚。研究影响 QE 及稳定性的根本原因, 探索受 潮及辐射老化深层次的物理机制, 对当前 CsI 光阴 极的研究和应用具有极为重要的意义。随着研究的 不断深入，更多提高 QE、防止或减缓光阴极老化的 工艺和方法也将会出现。作者认为, CsI 薄膜光阴极 下一步的研究方向与研究重点可能在以下几个方面:
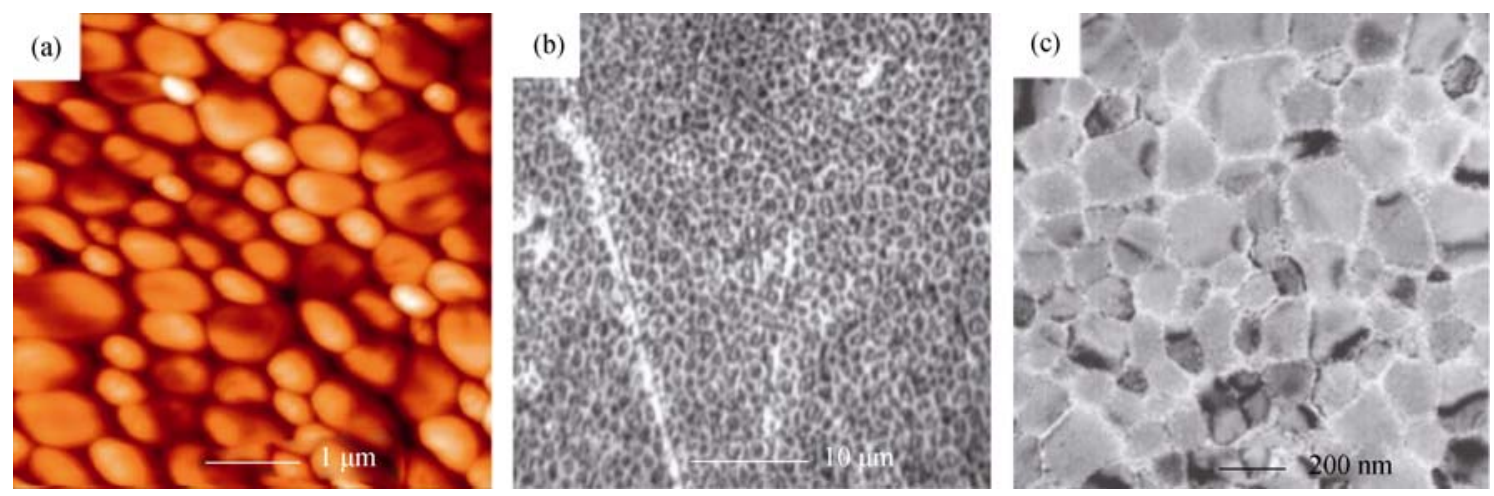

图 4 (a) $5 \mathrm{~d}$ UV 辐照后 CsI 薄膜的 AFM 照片 ${ }^{[11]}$; (b) $5 \mathrm{~d} \mathrm{UV}$ 辐照后的 PEEM 照片 ${ }^{[11]}$; (c)UV 辐照后的 TEM 照片 ${ }^{[48]}$

Fig. 4 (a) AFM image of the $5 \mathrm{~d}$ UV-irradiated CsI photocathode ${ }^{[11]}$; (b) PEEM image of the 5 d UV-irradiated CsI photocathode ${ }^{[11]}$; (c) TEM image of the UV-irradiated CsI film ${ }^{[48]}$ 
1) 对影响效率因素以及老化机理中某些还未 确定和尚有争义的问题进行更深入的研究, 并从原 理上突破，以指导制备方法和工艺的改进。

2) 发展微结构的基底, 提高 $\mathrm{QE}$ 和稳定性。虽然 早在 1994 年 $\mathrm{Lu}$ 等 ${ }^{[64]}$ 就从理论上预言了楔形微结构基 底可以增加 CsI 薄膜光阴极的 QE, 但人们对微结构基 底研究的实际步伐才刚刚开始。随着光刻技术与纳米 技术的飞速发展, 以及某些潜在基底材料表面微纳修 饰与改性研究的不断深入 ${ }^{[65]}$, 可以预测, 采用微结构 基底的 CsI 光阴极将在辐射探测领域大有作为。

3) 利用 CsI 薄膜的自组装性质, 通过纳米尺寸效 应和表面效应改进电子传输及光电发射性质。2011 年, Fedorov 等[66]首次在 $\mathrm{LiF}$ 基底上观察到了 CsI 薄膜的 固态去润湿现象, 展现了其在纳米改性方面的诱人发 展前景 ${ }^{[67]}$ 。目前, 对 CsI 薄膜自组装的研究才刚刚起 步 ${ }^{[68]}$, 而与 CsI 结构类似的 $\mathrm{CsCl}$ 薄膜与 $\mathrm{Au}$ 阴极薄膜 的自组装特性引起了研究者广泛关注 ${ }^{[69-70]}$ 。

4) 与其它材料组合, 改变某一波段的 QE 或实 现某些波段的带通选能。在激光等离子体研究中, 某些诊断设备的光阴极已通过多材料组合和改变占 空比等方式实现了带通选能 ${ }^{[71]}$, 而这也可能成为 CsI 薄膜光阴极研究的一个重要方向。

\section{参考文献:}

[1] FENG J, SHIN H J, NASIATKA J R, et al. An x-ray streak camera with high spatio-temporal resolution. Appl. Phys. Lett., 2007, 91(13): 134102-1-3.

[2] MOLNAR L. The ALICE HMPID detector ready for collisions at the LHC. Nucl. Instrum. Methods Phys. Res., Sect. A, 2008, 595(1): 27-30.

[3] NAPPI E. Trends in the development of large area photon detectors for Cherenkov light imaging applications. Nucl. Instrum. Methods Phys. Res., Sect. A, 2003, 504(1/2/3): 70-87.

[4] WANG W, FANG Z H, JIA G, et al. Multispectral X-ray imaging with a multichannel Kirkpatrick-Baez microscope for imploded core temperature observation. The European Physical Journal D, 2014, 68(5):129-1-5.

[5] XIE W Q, LI Y L, LI J, et al. Study of VUV Detector Based on Thinner THGEM for CDEX. Nuclear Science Symposium and Medical Imaging Conference (NSS/MIC), Anaheim, CA, 2012: 1127-1130.

[6] XIE Y G, ZHANG A W, LIU Y B, et al. Influence of air exposure on CsI photocathodes. Nucl. Instrum. Methods Phys. Res., Sect. A, 2012, 689: 79-86.

[7] TREMSIN A S, SIEGMUND O H. The Quantum Efficiency and Stability of UV and Soft X-ray Photocathodes. Proc. SPIE 5920,
Ultrafast X-Ray Detectors, High-Speed Imaging, and Applications, San Diego, USA, 2005: 592001-1-13.

[8] HOEDlmoser H, BRAEM A, De CATAldo G, et al. Long term performance and ageing of CsI photocathodes for the ALICE/HMPID detector. Nucl. Instrum. Methods Phys. Res., Sect. A, 2007, 574(1): 28-38.

[9] SINGH B K, TRILOKI, GARG P, et al. VUV-induced radiation ageing processes in CsI photocathodes studied by microscopy and spectroscopy techniques. Nucl. Instrum. Methods Phys. Res., Sect. A, 2009, 610(1): 350-353.

[10] SINGH B K, SHEFER E, BRESKIN A, et al. CsBr and CsI UV photocathodes: new results on quantum efficiency and aging. Nucl. Instrum. Methods Phys. Res., Sect. A, 2000, 454(2/3): 364-378.

[11] SINGH B K, NITTI M A, VALENTINI A, et al. Ageing of CsI thin film photocathodes induced by UV photons. Nucl. Instrum. Methods Phys. Res., Sect. A, 2007, 581(3): 651-655.

[12] BUZULUTSKOV A F. Gaseous photodetectors with solid photocathodes. Phys. Part. Nuclei, 2008, 39(3): 424-453.

[13] BRAEM A, DAVENPORT M, Di MAURO A, et al. Aging of large-area CsI photocathodes for the ALICE HMPID prototypes. Nucl. Instrum. Methods Phys. Res., Sect. A, 2003, 515(1): 307-312.

[14] PIUZ F. Ring Imaging CHerenkov systems based on gaseous photo-detectors: trends and limits around particle accelerators. Nucl. Instrum. Methods Phys. Res., Sect. A, 2003, 502(1): 76-90.

[15] NAPPI E. CsI RICH detectors in high energy physics experiments. Nucl. Instrum. Methods Phys. Res., Sect. A, 2001, 471(1): 18-24.

[16] SCHYNS E. Status of large area CsI photocathode developments. Nucl. Instrum. Methods Phys. Res., Sect. A, 2002, 494(1/2/3): 441-446.

[17] XIE Y G, LIU H B, ZHANG A W, et al. Quantum efficiency measurement of CsI photocathodes using synchrotron radiation at BSRF. Nucl. Instrum. Methods Phys. Res., Sect. A, 2012, 664(1): 310-316.

[18] NITTI M A, NAPPI E, VALENTINI A, et al. Progress in the production of CsI and diamond thin film photocathodes. Nucl. Instrum. Methods Phys. Res., Sect. A, 2005, 553(1/2): 157-164.

[19] NITTI M A, VALENTINI A, SENESI G S, et al. Ion-beam sputtering deposition of CsI thin films. Applied Physics A, 2005, 80(8): 1789-1791.

[20] FAIRCHILD S B, BACK T C, MURRAY P T, et al. Low work function CsI coatings for enhanced field emission properties. $J$. Vac. Sci. Technol. A, 2011, 29(3): 031402-1-6.

[21] BRENDEL' V M, BUKIN V V, GARNOV S V, et al. Fabrication of alkali halide UV photocathodes by pulsed laser deposition. Quantum Electron., 2012, 42(12): 1128-1132.

[22] BUZULUTSKOV A, BRESKIN A, CHECHIK R. Heat enhancement of the photoyield from CsI, NaI and CuI photocathodes. Nucl. 
Instrum. Methods Phys. Res., Sect. A, 1995, 366(2/3): 410-412.

[23] HOEDlmoser H, BRAEM A, De CATAldo G, et al. Production technique and quality evaluation of CsI photocathodes for the ALICE/HMPID detector. Nucl. Instrum. Methods Phys. Res., Sect. A, 2006, 566(2): 338-350.

[24] BRESKIN A. CsI UV photocathodes: history and mystery. Nucl. Instrum. Methods Phys. Res., Sect. A, 1996, 371(1/2): 116-136.

[25] FRIESE J, GERNHÄUSER R, HOMOLKA J, et al. Enhanced quantum efficiency for CsI grown on a graphite-based substrate coating. Nucl. Instrum. Methods Phys. Res., Sect. A, 1999, 438(1): 86-93.

[26] VALENTINI A, NAPPI E, NITTI M A. Influence of the substrate reflectance on the quantum efficiency of thin CsI photocathodes. Nucl. Instrum. Methods Phys. Res., Sect. A, 2002, 482(1/2): 238-243.

[27] LU C, MCDONALD K T. Properties of reflective and semitransparent CsI photocathodes. Nucl. Instrum. Methods Phys. Res., Sect. A, 1994, 343(1): 135-151.

[28] BOUTBOUL T, BRESKIN A, CHECHIK R, et al. On the surface morphology of thin alkali halide photocathode films. Nucl. Instrum. Methods Phys. Res., Sect. A, 1999, 438(2/3): 409-414.

[29] LI Y K, CHEN T, DENG B, et al. Energy spectral response of photocathode for soft X-ray streak camera. High Power Laser and Particle Beams, 2014, 26(02): 02202-1-4.

[30] YUAN Z, ZENG P, DENG B, et al. Spectral Sensitivity Calibration of $\mathrm{Au}$ and CsI Photocathodes of High Speed X-ray Scanning Camera. Proc. SPIE 8419, 6th International Symposium on Advanced Optical Manufacturing and Testing Technologies: Optoelectronic Materials and Devices for Sensing, Imaging, and Solar Energy, Xiamen, China, 2012: 841921-1-6.

[31] ZENG P, YUAN Z, DENG B, et al. Spectral response calibration of $\mathrm{Au}$ and CsI transmission photocathodes of X-ray streak camera in a 60-5500 eV photon energy region. Acta Phys. Sin. 2012, 61(15): 379-385.

[32] BRAEM A, JORAM C, PIUZ F, et al. Technology of photocathode production. Nucl. Instrum. Methods Phys. Res., Sect. A, 2003, 502(1): 205-210.

[33] LI M, NI Q L, CHEN B. Calculation of quantum efficiency of alkali halide photocathode materials in the extreme ultraviolet region. Acta Phys. Sin., 2009, 58(10): 6894-6901.

[34] LARRUQUERT J I, MENDEZ J A, AZNAREZ J A, et al. Optical properties and quantum efficiency of thin-film alkali halides in the far ultraviolet. Appl. Optics, 2002, 41(13): 2532-2540.

[35] [35] GARAI B, RADHAKRISHNA V, RAJANNA K. Effect of vacuum treatment on CsI photocathode performance in UV photon detectors. Optical Materials Express, 2013, 3(7): 948-953.

[36] MAURO A D, MARTINENGO P, PIUZ F, et al. Study of the quantum efficiency of CsI photocathodes exposed to oxygen and water vapour. Nucl. Instrum. Methods Phys. Res., Sect. A, 2001, 461(1/2/3): 584-586.

[37] NITTI M A, CIOFFI N, NAPPI E, et al. Influence of bias voltage on the stability of CsI photocathodes exposed to air. Nucl. Instrum. Methods Phys. Res., Sect. A, 2002, 493(1/2): 16-24.

[38] KUMAR K, ARUN P, KANT C R, et al. The effect of cesium metal clusters on the optical properties of cesium iodide thin films. Applied Physics A, 2010, 99(1): 305-310.

[39] KUMAR K, ARUN P, KANT C R, et al. Metal cluster's effect on the optical properties of cesium bromide thin films. Appl. Phys. Lett., 2012, 100(24): 243106-1-7.

[40] TIAN J Q, JIANG D L, SUN X P, et al. Study on new MCP reflection X-ray sensitive film of variable density halide. Chinese Journal of Luminescence, 2002, 23(5): 513-517.

[41] NITTI M A, TINTI A, VALENTINI A, et al. Influence of the substrate surface texture on the photon-sensitivity stability of CsI thin film photocathodes. Nucl. Instrum. Methods Phys. Res., Sect. A, 2009, 610(1): 234-237.

[42] SHEFER E, BRESKIN A, BOUTBOUL T, et al. Photoelectron transport in CsI and CsBr coating films of alkali antimonide and CsI photocathodes. J. Appl. Phys., 2002, 92(8): 4758-4771.

[43] SINGH B K, NAPPI E, NITTI M A, et al. Role of the substrate reflectance and surface-bulk treatments in CsI quantum efficiency. Nucl. Instrum. Methods Phys. Res., Sect. A, 2003, 502(1): 108-111.

[44] HALVORSON C, HOUCK T, MACPHEE A, et al. High energy photocathodes for laser fusion diagnostics. Rev. Sci. Instrum., 2010, 81(10): 10E309-1-3.

[45] LEE J, PARK T, LEE W, et al. Evaluation of a cesium iodide photocathode assisted with MgO-coated multiwall carbon nanotubes. Appl. Phys. Lett., 2010, 96(14): 141109-1-3.

[46] QIAN W J, LAI H W, PEI X Z, et al. Improving field emission by constructing CsI-AlN hybrid nanostructures. J. Mater. Chem., 2012, 22(35): 18578-18582.

[47] OPACHICH Y P, KALANTAR D H, MACPHEE A G, et al. High performance imaging streak camera for the National Ignition Facility. Rev. Sci. Instrum., 2012, 83(12): 125105-1-6.

[48] TREMSIN A S, RUVIMOV S, SIEGMUND O H W. Structural transformation of CsI thin film photocathodes under exposure to air and UV irradiation. Nucl. Instrum. Methods Phys. Res., Sect. A, 2000, 447(3): 614-618.

[49] FAN L, YANG Z W, CHEN T, et al. Influence of air exposure on the structure and properties of cesium iodide film. Acta Phys. Sin. 2014, 14(63):146801-1-7.

[50] TRILOKI, DUTTA B, SINGH B K. Influence of humidity on the photoemission properties and surface morphology of cesium iodide photocathode. Nucl. Instrum. Methods Phys. Res., Sect. A, 2012, 
695: 279-282.

[51] KUMAR K, ARUN P, KANT C R, et al. The effect of cesium metal clusters on the optical properties of cesium iodide thin films. Applied Physics A, 2010, 99(1): 305-310.

[52] ALMEIDA J, BRAEM A, BRESKIN A, et al. Microanalysis surface studies and photoemission properties of CsI photocathodes. Nucl. Instrum. Methods Phys. Res., Sect. A, 1995, 367(1/2/3): 337-341.

[53] BOUTBOUl T, AKKERMAN A, BRESKIN A, et al. Escape length of ultraviolet induced photoelectrons in alkali iodide and $\mathrm{CsBr}$ evaporated films: Measurements and modeling. J. Appl. Phys., 1998, 84(5): 2890-2896.

[54] TREMSIN A S, SIEGMUND O H. The Dependence of Quantum Efficiency of Alkali Halide Photocathodes on the Radiation Incidence angle. Proc. SPIE 3765, EUV, X-Ray, and Gamma-Ray Instrumentation for Astronomy X, Denver, 1999: 441-451.

[55] BUZULUTSKOV A, BRESKIN A, CHECHIK R. Photoemission from CsI/LiF and CsI/NaF films, enhanced by exposure to water vapour. Nucl. Instrum. Methods Phys. Res., Sect. A, 1996, 372(3): 572-574.

[56] SENESI G S, NITTI M A, VALENTINI A. A scanning electron and atomic force microscopy study of the surface morphology and composition of CsI films as affected by evaporation rate and humid-air exposure. Microsc. Microanal., 2005, 11(2): 124-132.

[57] NITTI M A, SENESI G S, LIOTINO A, et al. Influence of the film deposition rate and humidity on the properties of thin CsI photocathodes. Nucl. Instrum. Methods Phys. Res., Sect. A, 2004, 523(3): 323-333.

[58] TREMSIN A S, SIEGMUND O H W. UV radiation resistance and solar blindness of CsI and $\mathrm{KBr}$ photocathodes. IEEE Transactions on Nuclear Science, 2001, 48(3): 421-425.

[59] TREMSIN A S, PEARSON J F, NICHOLS A P, et al. $\mathrm{X}$-ray-induced radiation damage in CsI, Gadox, $\mathrm{Y}_{2} \mathrm{O}_{2} \mathrm{~S}$ and $\mathrm{Y}_{2} \mathrm{O}_{3}$ thin films. Nucl. Instrum. Methods Phys. Res., Sect. A, 2001, 459(3): 543-551.

[60] TREMSIN A S, SIEGMUND O H W. Quantum efficiency and stability of alkali halide UV photocathodes in the presence of elec- tric field. Nucl. Instrum. Methods Phys. Res., Sect. A, 2003, 504(1): 4-8.

[61] VA'VRA J. Physics and chemistry of aging-early developments. Nucl. Instrum. Methods Phys. Res., Sect. A, 2003, 515(1): 1-14.

[62] RUDOLF P, MARCHAL F, SPORKEN R, et al. Laterally resolved measurements of polycrystalline cesium iodide surfaces. Nucl. Instrum. Methods Phys. Res., Sect. A, 1997, 387(1/2): 163-170.

[63] TREMSIN A S, SIEGMUND O H W. Heat enhancement of radiation resistivity of evaporated CsI, KI and KBr photocathodes. Nucl. Instrum. Methods Phys. Res., Sect. A, 2000, 442(1/2/3): 337-341.

[64] LU C, MCDONALD K T. Properties of reflective and semitransparent CsI photocathodes. Nucl. Instrum. Methods Phys. Res., Sect. A, 1994, 343(1): 135-151.

[65] KAN T, MATSUMOTO K, SHIMOYAMA I. Nano-pattern Replication Using Parylene Thin Film for Optical Applications. Micro Electro Mechanical Systems, IEEE 20th International Conference on, Hyogo, 2007: 819-822.

[66] FEDOROV A, LEBEDINSKY A, MATEYCHENKO P. Dewetting behavior of CsI layers on LiF substrate. J. Cryst. Growth, 2011, 318(1): 595-598.

[67] THOMPSON C V. Solid-state dewetting of thin films. Ann. Rev. Mater. Res., 2012, 42: 399-434.

[68] YAO D L, GU M, LIU X L, et al. Performance of columnar CsI(Tl) scintillation films prepared on special pre-deposited layers. Appl. Surf. Sci., 2013, 276: 776-781.

[69] LIU J, ZHANG X S, DONG G Q, et al. The performances of silicon solar cell with core-shell pn junctions of micro-nano pillars fabricated by cesium chloride self-assembly and dry etching. Applied Physics A, 2014, 114(4): 1175-1179.

[70] WANG Y Y, GAO Y, WANG X M, et al. Fabrication and ultraviolet photoemission characteristics of novel Au photocathodes. High Power Laser and Particle Beams, 2013, 25(10): 2627-2630.

[71] GUO L, LI S W, ZHENG J, et al. A compact flat-response x-ray detector for the radiation flux in the range from $1.6 \mathrm{keV}$ to $4.4 \mathrm{keV}$. Meas. Sci. Technol., 2012, 23(6): 065902-1-6. 